$$
\operatorname{CONE}-931234--12
$$

\title{
Cellular Automata Modeling of Weld Solidification Structure*
}

\author{
W. B. Dress, Thomas Zacharia, and B. Radhakrishnan
}

Oak Ridge National Laboratory

\author{
To be published in the proceedings of the \\ International Conference on Modeling and \\ Control of Joining Processes \\ Orlando, Florida \\ December 8-10, 1993
}

\begin{abstract}
"The submitted manuscript has been authored by a contractor of the U. S. Government under contract No. DE-AC05$840 R 21400$. Accordingly, royalty-free license to publish or reproduce the published form of this contribution, or allow others to do so, for U. S. Government purposes."
\end{abstract}

\section{DISCLAIMER}

\begin{abstract}
This report was prepared as an account of work sponsored by an agency of the United States Government. Neither the United States Government nor any agency thereof, nor any of their employees, makes any warranty, express or implied, or assumes any legal liability or responsibility for the accuracy, completeness, or usefulness of any information, apparatus, product, or process disclosed, or represents that its use would not infringe privately owned rights. Reference herein to any specific commercial product, process, or service by trade name, trademark, manufacturer, or otherwise does not necessarily constitute or imply its endorsement, recommendation, or favoring by the United States Government or any agency thereof. The views and opinions of authors expressed herein do not necessarily state or reflect those of the United States Government or any agency thereof.
\end{abstract}

*Research sponsored by the U. S. Department of Energy, under contract DE-AC05-84OR21400 with Marin Marietta Energy Systems, Inc. 


\section{DISCLAIMER}

Portions of this document may be illegible in electronic image products. Images are produced from the best available original document. 


\title{
CELLULAR . ALTOMATA MODELING OF WELD SOLIDIFICATION STRUCTURE
}

\author{
W. B. Dress ${ }^{1}$, Thoomas Zacharia ${ }^{2}$, and B. Radhakrishnan ${ }^{2}$
}

\begin{abstract}
We explore the use of cellular autommata in modeling arc-welding processes. A brief discussion of cellular automata and their previouus use in micro-scale solidification simulations is presented. Macro-scale thermal calculations foor are-welding a thin plate are shown to give good quantitative and qualitative results. Combiningr the two calculations in a single cellular array provides a realistic simulation of grain growth 1 in a welding process. Results of simulating solidification in a moving melt pool in a poly-crystalliiine alloy sheet are presented.
\end{abstract}

\section{NTRODUCTION}

Melt-pool shape is a strong factor im the development of microstructures developing in the fusion zone of a weld. These structures pizay a major role in such defect formation as microporosity or hot-cracking and can affect solid-state transformations that may subsequently occur during cooling. In this sense, they are an iimportant step in any welding process prediction. This paper treats some of the fundamental asspects associated with weld-pool solidification. Included are such effects as heterogeneous nuccleation of grains in the bulk of the liquid, growth kinetics, preferential growth directions of cdendrites (as simulated by grain crystallographic axes), and competition between growth morphnologies.

The dominant methods for simulatuing joining processes are based on finite-element and finitedifference analysis. While these teechniques give excellent results on a macroscopic level, their application on a meso- or micro-scicale level, where grain structure effects are important, is not entirely satisfactory. The advent of cellular automata (CA) techniques in recent years may provide another method of efficiemtly simulating material behavior under various solidification and joining processes.

\section{CAS AS EFFICIENT MODELERS}

Although cellular automata have boeen used for many years as discrete, micro-scale simulators, we have begun to explore their usee in modeling meso-scale thermal and metallurgical problems with complicated boundary conditiions. The classical CA is an autonomous device having no coupling to the external world [1] : once its internal states are set and its rules chosen, it evolves as an independent system with eitther absorbing boundaries or within a toroidal (wrap-around) geometry. In an earlier paper, Dreess [2] showed how to modify conceptually the CA, allowing realistic boundaries for simulating :the physics of matter and energy exchange at these boundaries with an external system comprissed of fields and heat baths. In this sense, the method is

Instrumentation and Controls Division, COak Ridge National Laboratory, Oak Ridge, TN 37831-6011

2 Materials Process Modeling Group, Oak IFRidge National Laboratory, Oak Ridge, TN 37831-6140 
reminiscent of standard finite-element analysis, but allows a simple means of specifying complex boundary conditions, both temporal and spatial.

The recent set of papers by Rappaz and Gandin [3] and Gandin, Rappaz, and Tintillier [4] have shown the value of the $\mathrm{CA}$ for realistic simulations for micro-structure formation in solidification processes. Dress explored the use of CAs in heat-transfer problems in Ref. 2. The present work incorporates both the thermal and grain-growth CAs into a single simulation with the goal of extending the application of $\mathrm{CA}$ simulators to the dynamic welding process.

\section{Initial Simulation of Grain Micro Structure}

The present study attempts to model the fusion-zone structure in a linear, autogenous GTA weld. The orientation and configuration of the grains that grow at the trailing edge of the melt pool are of primary interest. The goal of this work is to develop a model that qualitatively and quantitatively reproduces what is seen in actual situations. Rappaz and Gandin (Ref. 3) simulated solidification in a casting by a CA. The authors showed that the grain growth dynamics are both qualitatively and approximately quantitatively correct for the case of solidification of an ingot.

A CA is specified by a set of states for each cell, a set of rules determining cell behavior, and a neighborhood function specifying which cells influence each other. The rules determine the transitions between states and are functions of the prior state and the neighborhood. In the microstructure simulation, the states were chosen to be a crystallographic orientation, a grain membership function, and a time of grain seeding. A reasonable model of crystal growth is to consider a wave of solidification spreading out from a seed or the boundary of a solidified grain, subject to the appropriate thermodynamic constraints. As pointed out in Ref. 3, this Huygen's Principle method can only reproduce the shapes having symmetry of the underlying CA lattice: squares for a rectangular lattice of cells, and hexagonal for a hexagonal lattice of cells. To achieve grain-shape independence from the $\mathrm{CA}$ lattice, we depart from the technique developed in Ref. 3 and define a "velocity density" or probability of cell attachment to a given grain that is based on a growth velocity as a function of the angle between the direction of the candidate CA cell in question and the crystallographic axis of the grain about to "attach" that cell. Although this is a departure from the method of Ref. 3 , it leads to results statistically indistinguishable from those therein, while maintaining the spirit of the CA computation of communication of information only between near neighbors.

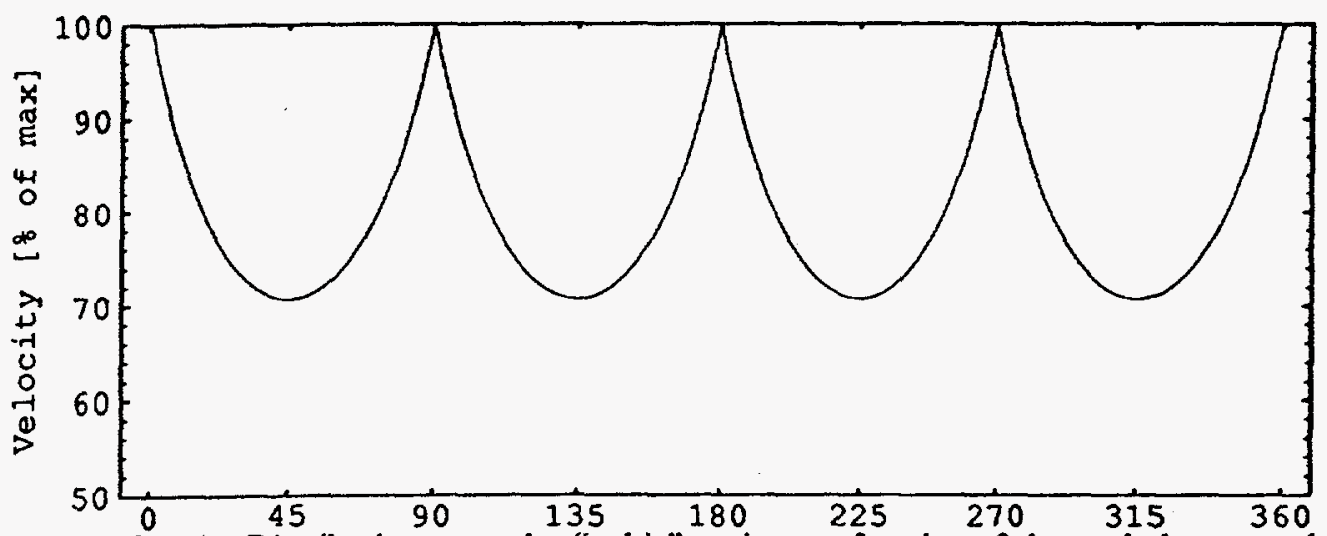

Figure 1. Velocity Distribution around a "cubic" grain as a function of the angle between the crystallographic axis and an arbitrary point. Note the four-fold symmetry about the 90 -degree points. The ordinate is in degrees, while the coordinate is in \% of the grain tip velocity. 
The velocity of the front spreading out from a grain boundary is proportional to the probability that a grain adds a cell located on the boundary at an angle $\alpha$ with respect to the principal axes of the grain. If we assume that the tip grows at the maximum velocity, and that the velocity 45 degrees from the tip grows at a rate proportional to $\cos (45 \mathrm{deg})$, we obtain the velocity a distribution proportional to $1 /(\cos \alpha+\sin \alpha)$. By taking $\alpha$ modulo 90 degrees, a four-fold grain symmetry is maintained. This form obviously neglects the microprocesses of dendritic growth and concentrates on the grain as the entity of physical importance. While not strictly correct, this simplifying assumption allows reasonable qualitative results to be obtained in meso-scale simulations. The velocity distribution around a cubic grain is shown in Figure 1 . The [100] axis is assumed to lie along the zero-degree direction.

\section{Equiaxed Grain Growth}

This velocity distribution was used to compute an initial grain structure for the melting and solidification simulation. The method follows that of Ref. 3 in that a certain undercooling is assumed, resulting in a tip velocity of about $0.25 \mathrm{~mm} / \mathrm{s}$. The initial grain structure was obtained by seeding growth sites with randomly chosen locations and orientations throughout the plate and running the $\mathrm{CA}$ forward in time until all the cells were attached to a grain. Where a CA cell was adjacent to two or more grains, a competition based on the time for the growth front to reach that cell determined to which grain it belonged. A typical (isolated) grain is shown in Figure 2.

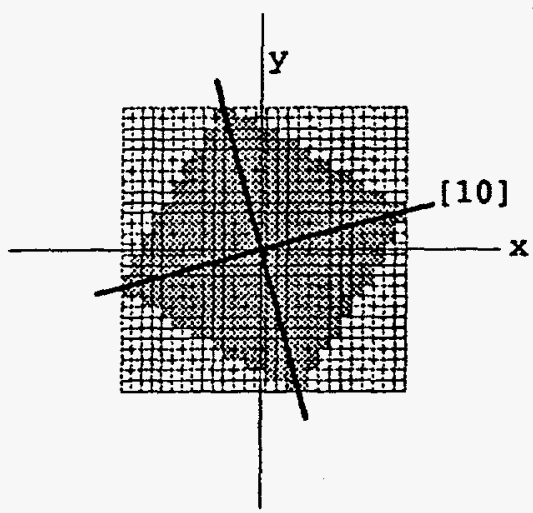

Figure 2. Growth of a single grain with [100] axis at an angle of 15 degrees with respect to the $x$ direction. The crystalline axes are shown as heavy lines centered on the grain. The superimposed grid depicts the location of the CA cells. Assuming a 50 micron cell size, this grain has dimensions $1 \mathrm{~mm}$ on a side.

Subsequent simulation of a cooling ingot with physical size, cell size, and undercooling specified as in Ref. 3 resulted in a close reproduction of the results shown therein, [Dress and Zacharia, Unpublished work]. This method successfully simulates both equiaxed and columnar grain growth. The success of the CA in thermal calculations, as developed in Ref. 2, and simulation of a cooling ingot with both columnar and equiaxed grain growth provides an impetus for attempting a simulation of solidification micro structure in an arc-welding problem. The $\mathrm{CA}$ appears to be a useful tool for a certain class of metallurgical problems.

In the next section, we briefly review the results of thermal calculations as carried out by a coarse-cell CA and then turn to the method and results of simulating grain growth in the weldfusion zone as calculated by a fine-cell $\mathrm{CA}$. 
A CA array in two or three dimensions can simulate the entire welding process of an arc traveling aluong a plate, melting the material to a certain depth as it moves. Each cell in the array must be asssigned an appropriate set of states that represent the physical properties of the material comprising:- the plate. The CA rules are particular discrete versions of the appropriate physical laws of heaat transfer and enthalpy. Those cells having a face on the plate boundary must be able to exchangee energy with the plate's environment.

\section{Thermal SBimulation}

Consider a:tgas-tungsten arc welding of a thin sheet as described by Zacharia, et al [5]. The CA model usead in this simulation is an array of 30 by 60 cells having dimensions $1 \mathrm{~mm}$ by $1 \mathrm{~mm}$ in the plane off the plate; the plate is $5 \mathrm{~mm}$ thick represented by one cell as we are considering only a two-dimeensional model at this time. The simulated heat source specification, taken from Ref. 5 , was Gaussiian in shape with a circular cross section delivering $95 \%$ of its power within a $3 \mathrm{~mm}$ radius. The simulated arc delivered $150 \mathrm{~A}$ at $15.1 \mathrm{~V}$ with $60 \%$ efficiency and traveled at a rate of 0.423 mmiss.

The resuitss of this simulation are shown in Figure 3 as a contour plot of isotherms within the plate some -33 seconds after the arc crossed the left edge.

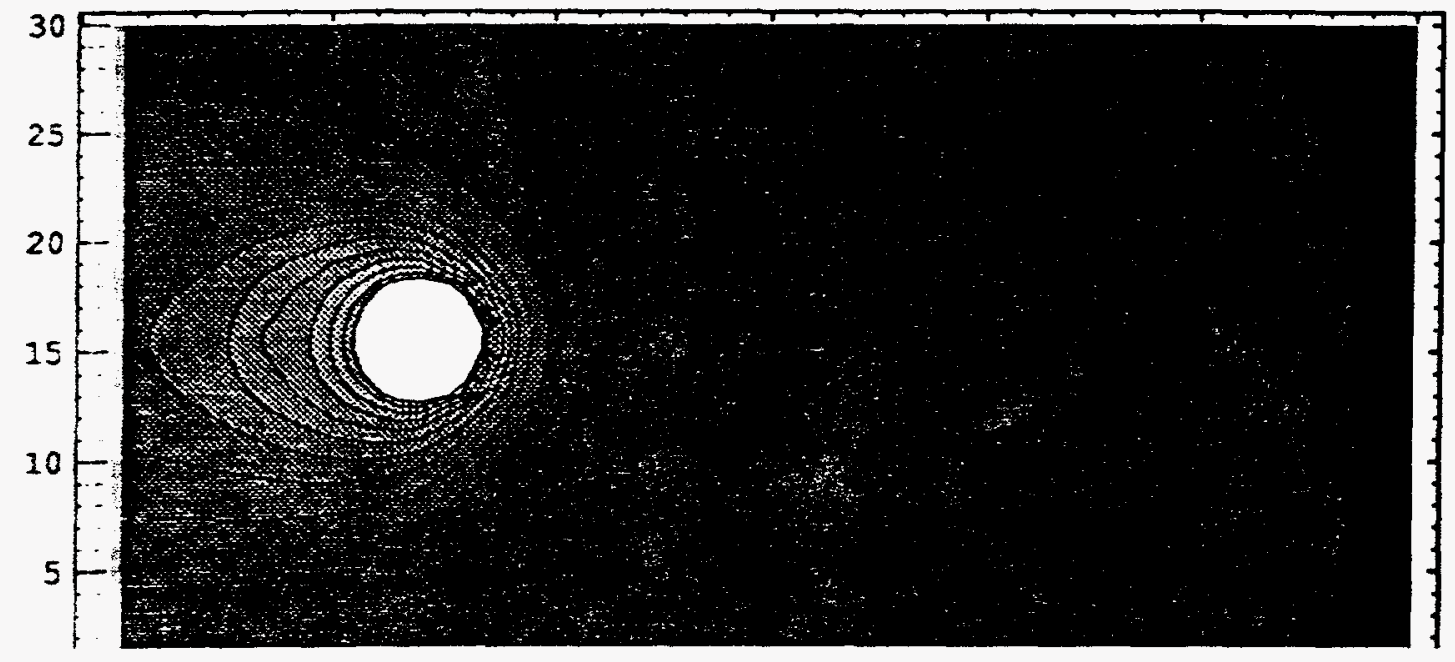

Figure 3. Contour plot of isotherms $33 \mathrm{sec}$ after starting the are off the plate to the left. The comrtours range from $200 \mathrm{C}$ to $2200 \mathrm{C}$ in steps of $200 \mathrm{C}$. The solidus isotherm is the one at $1400 \mathrm{C}$ (thind in from the outer fully closed contour). Coordinates are in $\mathrm{mm}$, and CA cells are $1 \mathrm{~mm}$ by 1 mmin. The boundary of white central disk indicates the $3 \mathrm{~mm}$ radius of the arc. Units are in $\mathrm{mm}$.

\section{Decoupling The Thermal Calculations}

Although mnot necessary, the thermal calculation was decoupled from the grain growth calculation to provide:-greater flexibility in exploring the effect of various rules and neighborhoods on grain growth pautterns. This was accomplished by deriving an approximate form for the solid-liquid interface. The results the thermal simulation were used as an external driving field that was 
ccoupled to the grain-growth $\mathrm{CA}$, and any consistent set of thermal cealculations may be used as input to the grain-growth CA.

A solidus isotherm was fit to a fourth-order polynomial to approximate the shape of that curve. The results of this fit are shown in Figure 4. A description of the: isotherm as a polynomial allowed both arbitrary scaling and arbitrary sampling, as well as pproviding a time-dependent critical solidus line that could be swept along any plate of arbitrary leength modeled by a CA grid of arbitrary cell size. In this way, the results of the thermal CA seeried as input to the graingrouth $\mathrm{CA}$, decoupling the two calculations.

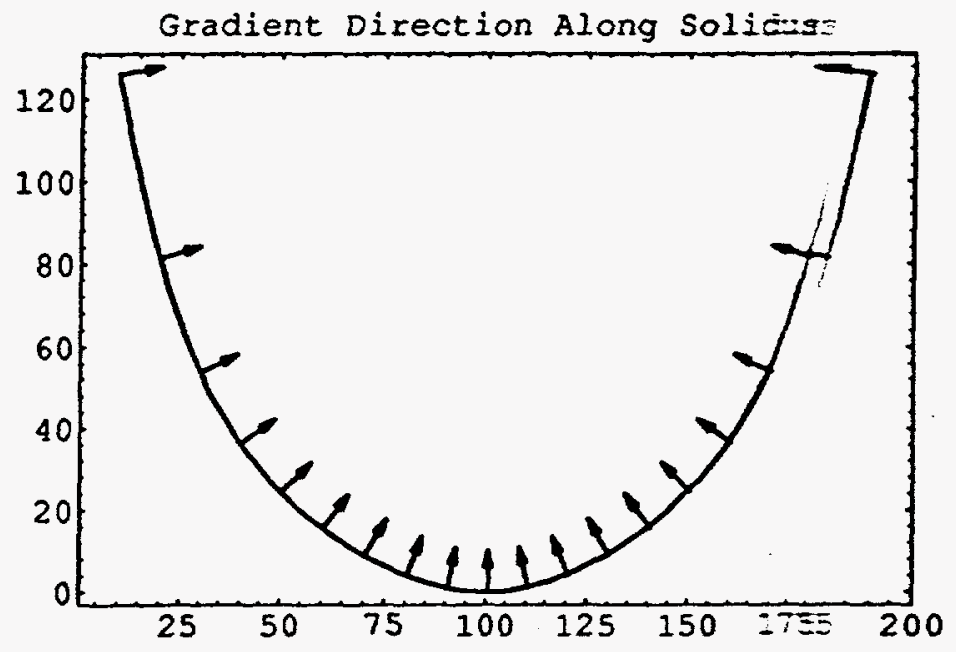

Figure 4. An isotherm at the solidus interface showing the direction of thes temperature gradient at every 10th cell along the width ordinate. The direction of arc travel is unpuards in this view; the centered vertical arrow indicates the unit vector in the direction of ar imotion. The coordinates refer to CA cells in the array.

Once the isotherm function has been determined for a particular pilate geometry, it is a simple matter to sample the function for different cell sizes and to computerthe thermal gradient at each cell site. Thus, both the cells leaving the melt pool and the locail interface velocity can be computed as a function of time. If $y_{T}(x, 0)$ is the polynomial expresssion for the position of the isotherm as a function of distance across the plate where $x$ is the :ordinate in Fig. 4, then the position of the isotherm at time $t$ is simply

$$
y_{T}(x, t)=y_{T}(x, 0)+v_{\text {arc }} t .
$$

Once the moving interface has been defined, it is used to determinae those cells changing from liquid to solid during any given time step. The method requires a chcoice of time step that is small enough so that only a few cells have crossed the interface at any particular instant. The criterion for cell selection is to choose only those cells whose center positicon as measured by $\mathbf{x}$ has just fallen beyond the $y_{T}(x, t)$ curve. Under the physical parameters mentioned above and with a 20 micron cell size, a time step of $10 \mathrm{~ms}$ results in approximately $15 \%$ of those cells lying on the solid-liquid interface being solidified at each step. These few cells then become candidates for attachment to existing grains. 


\section{Local Growth Directions \& Angles}

To attach the candidate cells to adjacent grains, we make use of the two dominant features of the welding process: heat-flow direction, and direction of arc travel. The direction of heat flow is assumed normal to the solid-liquid interface and is proportional to the gradient discussed above. New candidate cells can only be attached to existing grains on which they border. If a candidate cell touches only one grain, it will be attached to that grain during the current time step. Only in the case of a candidate cell bordering on two or more grains can a choice be made. The competition as to which grain attaches the candidate cell determines the crystalline growth pattern of the solidification. This competition is based on a probability of attachment calculated from the crystallographic direction, the heat-flow direction, and a direction of attachment.

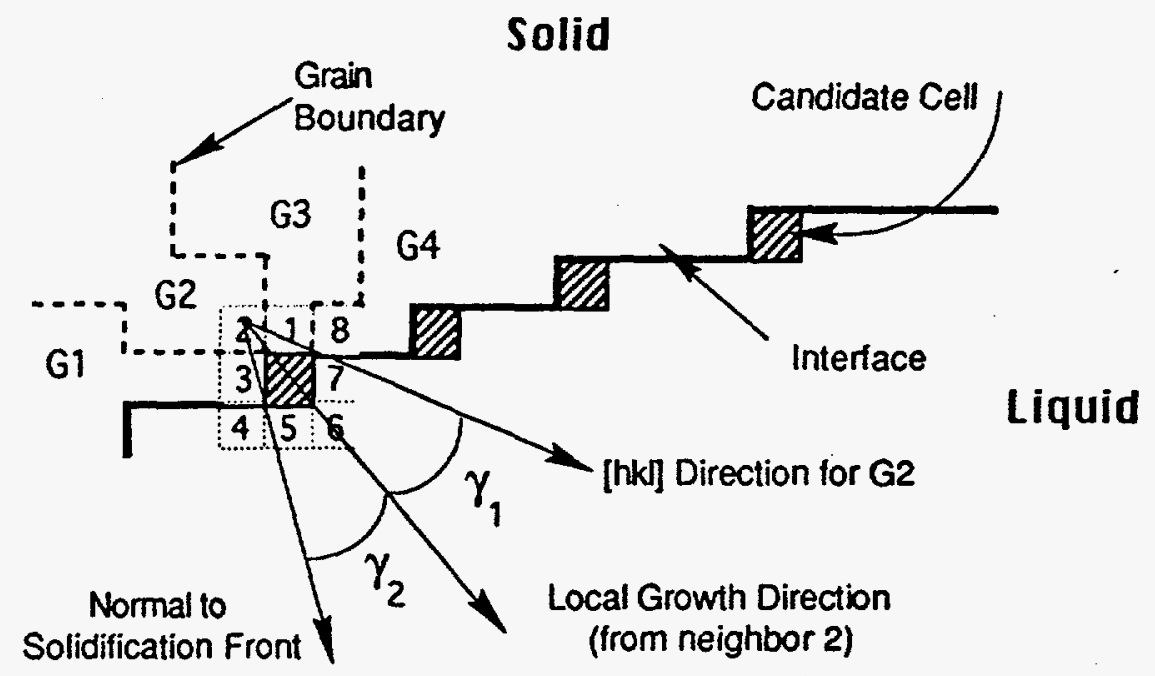

Figure 5. Cell attachment illustration. The candidate cells are shown with bar shading, and the solid-liquid interface as a heavy line. The cells are depicted as squares comprising the cellular array; which is not shown for clarity. The candidate cell discussed in the text is the one on the left, shown at the center of a neighborhood of CA cells. This candidate cell borders on four grains indicated as G1 through G4.

Consider the portion of the CA array illustrated in Figure 5. The candidate grains are indicated by the shaded squares and the solid-liquid interface by the heavy line. The arc is traveling to the right. In this figure, we are examining a section of the CA lying along the upper portion of the plate, somewhere behind the arc position.

To keep the interface at a constant shape, as determined by the moving curve given by of Eq. 1 , each of the candidate cells must solidify during the time step that they are produced by attaching to one of the neighboring grains. For each of the possible solid cells to which a candidate can attach, we define a local growth direction (direction of attachment) as a vector from the center of the solid cell in the neighborhood of the candidate grain through the center of the candidate grain itself. The local growth direction from neighbor 2 (lying in grain 2) is shown in the Fig. 5. Both of the geometrical directions - the gradient or normal to the interface, and the direction of the [100] crystallographic axis-are referred to this local growth direction, giving the angles labeled 
$\gamma_{1}$ and $\gamma_{2}$ in the figure. The only ambiguity is the choice for the crystallographic zairection of the grain in question (labeled [hkl] in the figure) as there are four possibilities duse to the cubic symmetry in the plane. We choose that axis lying closest to the local grouth direection; note that this direction is necessarily forward, in the direction of arc travel.

The probability that a candidate cell attaches to a parent grain is proportional ic: $\cos \gamma_{1} \cdot \cos \gamma_{2}$, where $\gamma_{1}$ is the angle between the local growth direction and the direction of maxximum growth denoted by [hkl], and $\gamma_{2}$ is the angle between the normal to the solidification froont and the local growth direction. This probability is calculated for each of the solid cells in the neighborhood, and the attachment of the candidate cell to a parent grain is made according to theese probabilities in the usual manner.

\section{RESULTS}

The grain structure resulting from a simulation of welding both single and poly-creystalline alloys shows patterns consistent with experimental data, suggesting that the model : is qualitatively correct within the approximations made. Figure 6 shows the results of one such ssimulation. The melting started about $2 \mathrm{~mm}$ from the left edge of the plate and the arc traveled ato the right at a velocity of $0.423 \mathrm{~mm} / \mathrm{s}$.

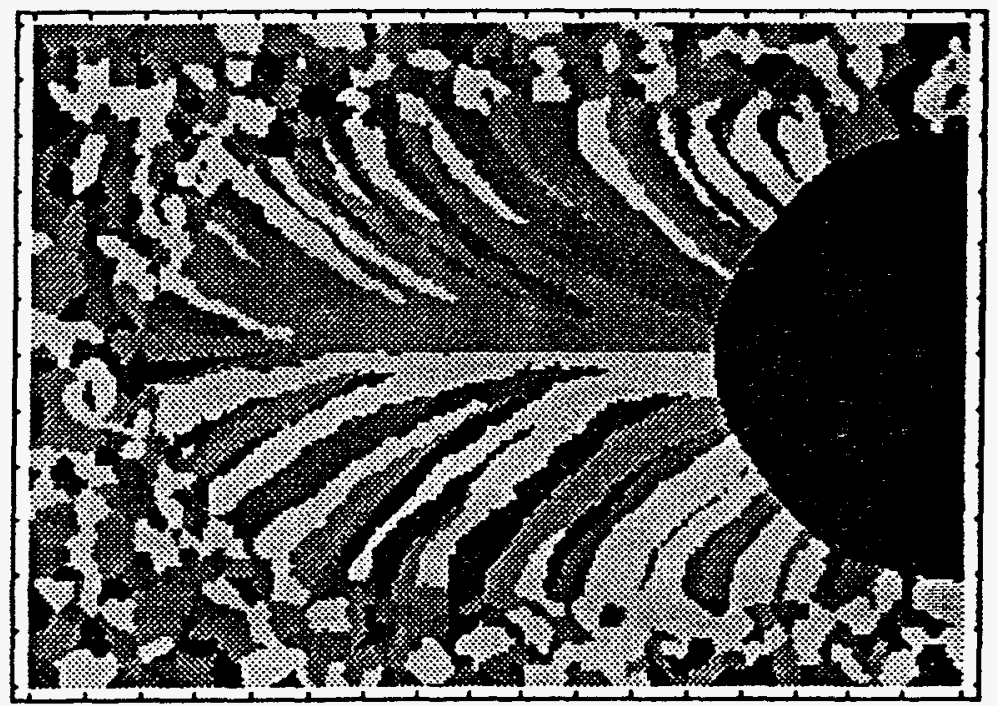

Figure 6 Partially solidified plate showing grain growth following the arc. Solidified grainns follow the thermal gradient and curve toward the arc position, about $3 \mathrm{~mm}$ off the figure to the right. The coordinate tick marks indicate plate dimensions in $\mathrm{mm}$.

While the thermal simulation was carried out for the plate shown in Fig. 1, only a central 12 by $17 \mathrm{~mm}$ region of that plate was used in the grain-growth simulations to reduce the number of inactive cells in the CA. The initial grain configuration was computed by sallowing 1500 randomly placed seeds to grow according to the method described in Section $\approx$ until each cell belonged to a grain. Each seed had a principal axis direction randomly chosen to ilie between -44 and +45 degrees ( -45 is equivalent to +45 given the four-fold symmetry) wittin respect to the horizontal (arc travel) direction. The integer angles were chosen with steps of cone degree. The 
size of each CA cell was 20 microns. resulting CA dimensions of 600 by 850 , or 510,000 cells in total.

\section{Discussion}

The random nature of the competitio ziliuded to above is evident in the curvature of the grains as solidifying cells are attached at the scoiidus ir:ierface. A "winner-take-all" competition would result in predominately vertically as $\dot{3}$ hrorizontally oriented grain boundaries, with any change of direction in growth taking place at a looses the competition due to the chamging gradient direction as the melt pool travels to the left. Since the winner is chosen in a probabuiistic fashion, certain grains also taper off in width as they gradually loose to a competitor. If places, this tapering off leads to the appearance of intermingling of two grains for shor dinstances. This effect is clearly visible the upper-left corner of the plate depicted in Fig. 6.

\section{Summary}

We have demonstrated that CAs are eficient and accurate simulators of the welding process for a thin plate. They reproduce the thermal behavior of material samples and show good results when simulating equiaxed and colummar grain grouth based on a growth-front distribution. The simulation of grain growth trailing a moving melt pool by CAs is both reasonable and efficient.

\section{ACEKNOWLEDGMENTS}

The research reported in this paper sass sponsored by the Division of Materials Sciences, United States Department of Energy under C.ontract No. DE-AC05-840R21400 with Martin Marietta Energy Systems, Inc.

\section{REFERENCES}

1. Tommaso Toffoli and Norman Margolus, Cellular Automata Machines, The MIT Press, Cambridge, MA, 1987.

2. W. B. Dress, "On Doing Science Bছ Computer," part of a Technical Report to be published by the U. S. Air Force, Wright-Pattersson AFB, Dayton, Ohio, 1993.

3. M. Rappaz and Ch.-A. Gandin, "Probabilistic Modelling of Microstructure Formation in Solidification Processes," Acta meatall. mater., 4(2), 345-60, 1993.

4. Ch.-A. Gandin, M. Rappaz, and R Tintillier, "Three-Dimensional Probabilistic Simulation of Solidification Grain Structures," Metall. Trans., 24A, 467-79, 1993

5. T. Zacharia, A. H. Eraslan, D. K. Aidun, and S. A. David, "Three-Dimensional Transient Model for Arc Welding Process," iMetall. Trans., 20B, 645-59, 1989. 